\title{
Energy shift and wave function overlap of metal-organic interface states
}

\author{
M. Marks, ${ }^{1}$ N. L. Zaitsev, ${ }^{2,3}$ B. Schmidt, ${ }^{1}$ C. H. Schwalb, ${ }^{1}$ A. Schölll, ${ }^{4}$ I. A. Nechaev, ${ }^{3,5}$ P. M. Echenique, ${ }^{2,6}$ \\ E. V. Chulkov, ${ }^{2,6}$ and U. Höfer ${ }^{1,2}$ \\ ${ }^{1}$ Fachbereich Physik und Zentrum für Materialwissenschaften, Philipps-Universität, D-35032 Marburg, Germany \\ ${ }^{2}$ Donostia International Physics Center (DIPC), E-20018 San Sebastián, Spain \\ ${ }^{3}$ Tomsk State University, 634050, Tomsk, Russia \\ ${ }^{4}$ Universität Würzburg, Experimentelle Physik VII and Röntgen Center (RCCM), D-97074 Würzburg, Germany \\ ${ }^{5}$ Nekrasov Kostroma State University, 156961 Kostroma, Russia \\ ${ }^{6}$ Departamento de Física de Materiales, UPV/EHU and CFM - MPC, Centro Mixto CSIC - UPV/EHU, E-20080 San Sebastián, Spain
}

(Received 20 June 2011; revised manuscript received 7 July 2011; published 11 August 2011)

\begin{abstract}
The properties of Shockley-type interface states between $\pi$-conjugated organic molecular layers and metal surfaces are investigated by time-resolved two-photon photoemission experiments and density functional theory. For perylene- and naphthalene-tetracarboxylic acid dianhydride (PTCDA and NTCDA) adsorbed on Ag(111), a common mechanism of formation of the interface state from the partly occupied surface state of the bare $\operatorname{Ag}(111)$ is revealed. The energy position is found to be strongly dependent on the distance of the molecular carbon rings from the metal and their surface density. Bending of the carboxyl groups enhances the molecular overlap of the interface state.
\end{abstract}

DOI: 10.1103/PhysRevB.84.081301

PACS number(s): 73.20.-r, 78.47.-p, 79.60.Dp

The energetic position and wave function overlap of electronic states at the interface between layers of organic molecules and metals is of fundamental interest for the design of organic semiconductor devices and for future applications of molecular electronics. Previous studies, both experimentally and theoretically, concentrated on those electronic states that either result directly from the chemical bonding at the interface or from the shift and broadening of localized molecular orbitals upon interaction with the metal substrate. ${ }^{1-8}$ However, also states intrinsic to metal surfaces are affected by adsorption and can become an important factor for the electronic coupling between metal and organic molecules. This has recently become apparent for 3,4,9,10-perylene-tetracarboxylicacid-dianhydride (PTCDA) on $\operatorname{Ag}(111)$, a structurally very well characterized model system for the interface between $\pi$-conjugated organic molecular layers and metals. ${ }^{4} \mathrm{~A}$ dispersing, free-electron-like electronic state located $0.6 \mathrm{eV}$ above the Fermi level was observed by tunneling spectroscopy ${ }^{9}$ and by two-photon photoemission (2PPE) ${ }^{10,11}$ It originates from the Shockley state of the $\operatorname{Ag}(111)$ surface which is upshifted from below the metallic Fermi level by as much as $0.7 \mathrm{eV}$. The initially partially occupied state becomes unoccupied and approaches the conduction band of the organic semiconductor. ${ }^{11-13}$

In this Rapid Communication we address the question which properties of the molecule-surface interaction determine the energetic position of such Shockley-type interface states (IS) and which factors facilitate a large overlap of the state with the molecular layer in order to tailor the degree of electronic coupling. For rare-gas adsorbates on noble metals it is well established that their adsorption also leads to an upshift of the Shockley state, albeit by much smaller values, and that the shift increases systematically from the weakly bound $\mathrm{Ar}$ to the more strongly interacting Xe. ${ }^{14,15}$ The comparison of our 2PPE experiments for 1,4,5,8-naphthalene-tetracarboxylic acid dianhydride (NTCDA) on $\mathrm{Ag}$ (111) with PTCDA/Ag(111) shows an analogous trend. The structurally similar, but smaller NTCDA molecules cause a smaller IS upshift than the larger
PTCDA molecules. The time-resolved 2PPE data indicate a similar overlap of the wave function with the metal in both cases. Density functional calculations explain the smaller shift by a larger distance of the NTCDA carbon plane from the surface. Surprisingly, in the metastable disordered PTCDA phase, when the molecules interact more strongly with the substrate, ${ }^{16}$ we observe a smaller and not a larger IS upshift. Lifetime measurements indicate a reduced overlap of the wave function with the metal. Theory relates this finding to the bending of the carboxyl groups which enables the IS wave function to extend more into the molecule.

The experiments have been conducted under ultra-high vacuum conditions using a $2 \mathrm{PPE}$ setup described previously. ${ }^{17}$ The photon energies of the blue and ultraviolet (UV) laser pulses were 3.10 and $4.70 \mathrm{eV}$ with pulse lengths of 47 and $70 \mathrm{fs}$, respectively. The overall energy resolution of the setup was $65 \mathrm{meV}$, the angular resolution $1.2^{\circ}$. Thin organic films of temperature gradient sublimated PTCDA and NTCDA were deposited onto sputter/annealed, clean $\operatorname{Ag}(111)$ surfaces at a rate of $0.2-0.4 \mathrm{ML} / \mathrm{min}$. Thermal desorption of multilayer films resulted in a commensurate monolayer for PTCDA ${ }^{4}$ and the relaxed monolayer for NTCDA. ${ }^{20}$ Sample cooling to $90 \mathrm{~K}$ was performed rapid enough ( $\gtrsim 15 \mathrm{~K} / \mathrm{min}$ ) to suppress the inverse melting phenomenon for NTCDA. ${ }^{18}$ The PTCDA-LT phase was prepared by adsorption at a sample temperature of $90 \mathrm{~K}$.

The DFT calculations have been performed using the pseudopotential method as implemented in OPENMX code within the local density approximation for the exchangecorrelation potential. ${ }^{12,19} \mathrm{~A}$ slab containing a nine-layer silver film was employed. The relaxed NTCDA monolayer was attached on one side of the film, the vacuum region was chosen to correspond to six silver interlayer spacings. The resulting periodic supercell has a rectangular unit cell of $11.56 \times$ $15.02 \AA^{2}$ and contains 216 silver atoms and 48 atoms belonging to two NTCDA molecules..$^{20}$ The surface Brillouin zone (SBZ) was sampled with a $3 \times 3 \mathbf{k}$-point mesh.

Figures 1(a) and 1(b) display the results of angleresolved 2PPE spectroscopy performed with a single-color 

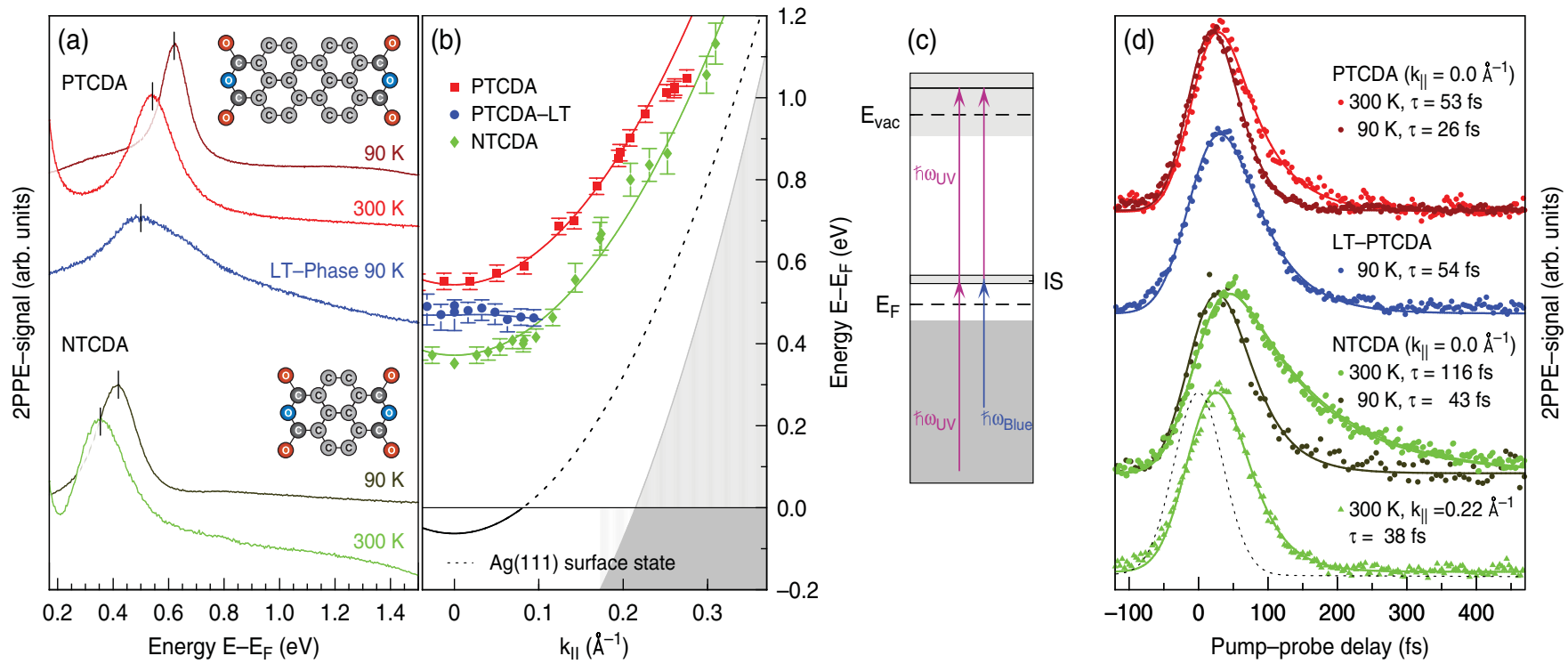

FIG. 1. (Color online) Energy-, angle- and time-resolved 2PPE data of monolayer films of NTCDA, PTCDA on Ag(111). (a) Normal emission (UV + UV) 2PPE spectra of commensurate PTCDA and NTCDA monolayers recorded at 300 and $90 \mathrm{~K}$ as indicated, and of the disordered LT-phase of PTCDA at $90 \mathrm{~K}$. The insets sketch the molecular structure for PTCDA (top) and NTCDA (bottom). (b) Angular dependence of the peak maxima of the interface state of the ordered PTCDA (squares, red) and NTCDA (diamonds, green) phases measured at $300 \mathrm{~K}$ and the PTCDA low-temperature phase (dots, blue) measured at $90 \mathrm{~K}$ together with projected $\mathrm{Ag}(111)$ bulk bands (gray shaded area) and the Shockley state of the clean surface (black). (c) Excitation schemes employed to 2PPE spectroscopy (left) and time-resolved 2PPE (right). (d) Time-resolved 2PPE data recorded at the peak maxima of the interface states of the different PTCDA and NTCDA monolayers. Lifetimes were extracted from best fits of a rate equation model using Gaussian laser pulses that reproduce the measured (blue + UV) cross correlation on the clean surface (dashed line).

$(\mathrm{UV}+\mathrm{UV})$-excitation scheme [cf. Fig. 1(c)]. In agreement with previous results, the well-ordered herringbone phase of PTCDA/Ag(111) exhibits a strongly dispersing state $\sim 0.6-1.0 \mathrm{eV}$ above the Fermi level $\left(E_{\mathrm{F}}\right) .{ }^{11}$ For one monolayer the band minimum at $k_{\|}=0$ is $E-E_{\mathrm{F}}=0.57 \pm 0.02 \mathrm{eV}$ at room temperature. Its energy increases slightly when additional layers are adsorbed or when the sample is cooled to liquid nitrogen temperature. ${ }^{17}$ Similar to PTCDA, the smaller NTCDA molecules can be adsorbed in a commensurate monolayer on $\mathrm{Ag}(111){ }^{20} \mathrm{We}$ observe an unoccupied dispersing interface state also for this system [green lines and data points in Figs. 1(a) and 1(b)]. It is located $0.2 \mathrm{eV}$ closer to the Fermi level $\left(E-E_{\mathrm{F}}=0.38 \pm 0.02 \mathrm{eV}\right.$ at $\left.k_{\|}=0\right)$ than the IS of PTCDA. The effective masses at the $\bar{\Gamma}$ point of both IS are similar (PTCDA: $0.46 \pm 0.1 m_{\mathrm{e}}$, NTCDA: $0.47 \pm 0.1 m_{\mathrm{e}}$ ) and both values are close to that of the Shockley state of the clean metal $^{15}\left(0.42 m_{\mathrm{e}}\right)$.

The results of the time-resolved 2PPE experiments [Fig. 1(d)] underline the similarities between the IS of the commensurate PTCDA and NTCDA layers. For these experiments we made use of a two-color (blue $+\mathrm{UV}$ )-excitation scheme. The unoccupied IS is populated by an intense blue pump pulse and the transient population is photoemitted by a weaker UV pulse with variable time delay [Fig. 1(c)]. ${ }^{21}$ The lifetime of $53 \pm 3$ fs determined for the IS of the PTCDA herringbone phase at $300 \mathrm{~K}$ and $k_{\|}=0$ agrees well with our previous results. ${ }^{11}$ For NTCDA we measure a considerably longer lifetime of $115 \pm 10 \mathrm{fs}$.

Before comparing these two values we note that both IS are located in the gap of the projected bulk bands of $\mathrm{Ag}(111)$.
Electrons excited into these states will decay inelastically by electron-hole-pair creation at a rate determined by the wave function overlap with the metal, the screening of the Coulomb interaction, and the available phase space in the metal. ${ }^{22}$ Under the realistic assumption of a similar screened Coulomb interaction at both interfaces (NTCDA/Ag and PTCDA/Ag) and taking into account the different phase-space factors for the decay at the different energies above the Fermi level, the measured lifetimes of the IS allow us to compare their overlap with the metal. In fact, the ratio of the lifetimes $\tau_{\mathrm{NTCDA}} / \tau_{\mathrm{PTCDA}}[116 / 53=2.19]$ is very close to the inverse phase space factor $\left(E_{\mathrm{PTCDA}}-E_{\mathrm{F}}\right)^{2} /\left(E_{\mathrm{NTCDA}}-E_{\mathrm{F}}\right)^{2}$ $\left[(0.57)^{2} /(0.38)^{2}=2.25\right]$ of a free electron gas close to the Fermi level. ${ }^{23}$ This suggests a similar overlap of both states with the metal. We arrive at the same conclusion when we compare the lifetimes of the interface states of NTCDA and PTCDA along their dispersing bands. ${ }^{24}$ At $k_{\|}=0.22 \AA^{-1}$, for example, the NTCDA-IS has a similar energy as the PTCDA-IS at $k_{\|}=0.19 \AA^{-1}\left(E-E_{\mathrm{F}} \simeq 0.8 \mathrm{eV}\right)$ and its lifetime [38 fs, Fig. 1(d)] is close to that of the PTCDA-IS ${ }^{17}$ (33 fs).

Our results thus indicate that the commensurate NTCDA and PTCDA layers have a qualitatively very similar influence on the Shockley surface state of $\operatorname{Ag}(111)$, except for a larger upshift induced by the more strongly interacting PTCDA.

This picture changes when we turn to the results obtained for the low temperature phase of PTCDA. Adsorption at sample temperatures below $150 \mathrm{~K}$ leads to an incommensurate PTCDA monolayer in which the molecules form dendritic islands. The reduced lateral interaction of the molecules in this phase goes along with a stronger binding to the surface. ${ }^{16}$ 


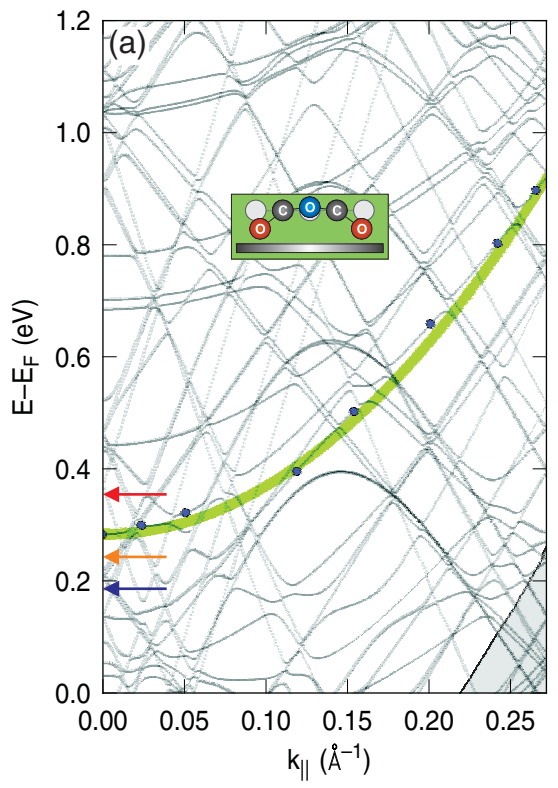

(b)

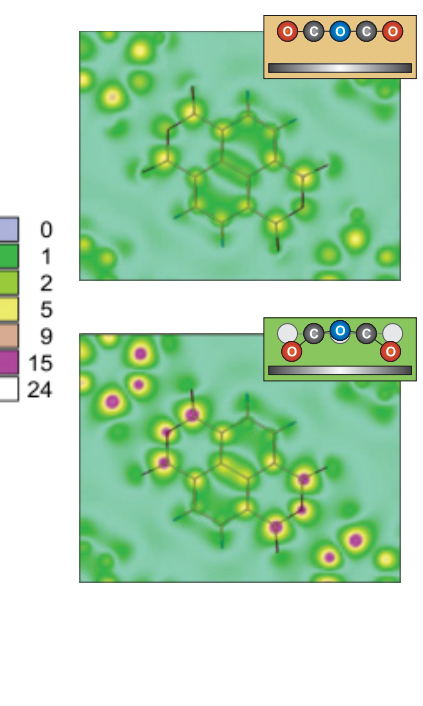

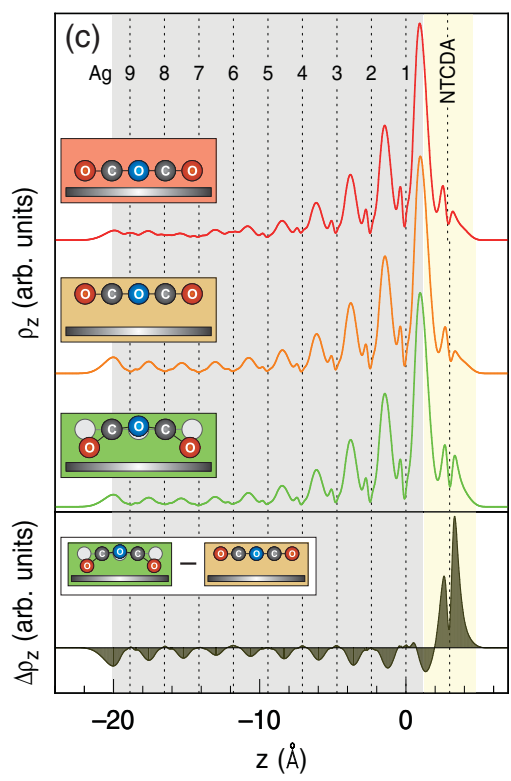

FIG. 2. (Color online) (a) Band structure of the NTCDA monolayer on the nine-layer $\operatorname{Ag}(111)$ film in the $\bar{\Gamma} \rightarrow \bar{X}_{1}$ direction of the surface Brillouin zone for the bend molecular geometry. Filled circles indicate wave vectors $\mathbf{k}$, at which the spatial distribution of the IS wave function was analyzed, the thick line represents the parabolic approximation of the corresponding dispersion, arrows indicate IS energies at $\bar{\Gamma}$ for different geometries (from top to bottom: red: $d=2.85 \AA$ flat, orange: $d=2.997 \AA$ flat, blue: less dense packing). (b) Spatial distribution of the IS probability density $\rho_{x y}$ at the $\bar{\Gamma}$ point in the vicinity of the molecular plane for the flat (top) and the bent-down geometry (bottom). (c) IS probability density $\rho_{z}$ perpendicular to the surface, $x y$ averaged over the unit cell (top: $\rho_{z}$ for the three considered NTCDA geometries; bottom: difference $\Delta \rho_{z}$ between the bent and the flat geometry at the distance of $2.997 \AA$ ).

Surprisingly, this stronger binding is not accompanied by a larger IS upshift as compared to the commensurate PTCDA phase. It is observed at an energy of $0.50 \pm 0.03 \mathrm{eV},{ }^{25}$ that is, $0.07 \mathrm{eV}$ lower than the IS of the more weakly bound commensurate phase [Fig. 1(a)]. The electron lifetime is $54 \pm 3 \mathrm{fs}$ [Fig. 1(d)]. Since the IS lifetime of the commensurate PTCDA phase is strongly temperature dependent, ${ }^{17}$ we have to compare this value, obtained at $90 \mathrm{~K}$, with lifetime measurements of the commensurate phases performed at the same temperature [Fig. 1(d)]. The lifetime of $54 \pm 3$ fs is almost twice the $26 \mathrm{fs}$ measured for commensurate PTCDA at $90 \mathrm{~K}$ and even longer than the IS lifetime of NTCDA (43 fs at $90 \mathrm{~K}$ ) though the latter state is closer to the Fermi level. Following the arguments above, the time-resolved experiments thus indicate that the wave function of the IS of the low-temperature PTCDA phase has less overlap with the metal than that of the ordered PTCDA and NTCDA layers.

In order to obtain a microscopic understanding of the origin of the observed energy shifts of the IS and its wave function overlap, we performed DFT calculations for three different geometries of the NTCDA monolayer. First, the molecules stay in their flat gas phase geometry, while they are artificially kept at the experimentally determined adsorption distance of the carbon plane $(d=2.997 \AA)$. Second, the flat molecules are moved slightly closer to the surface $(d=2.85 \AA)$, that is, to the adsorption distance of the ordered PTCDA monolayer. ${ }^{16}$ Third, the experimental adsorption geometry of NTCDA with bent carboxylic oxygen atoms is simulated $(d=2.997 \AA) .{ }^{26}$ Comparing calculations for one molecule (NTCDA) in different geometries allows us to pinpoint the relevant factors that determine properties of the interface state very clearly, although we can of course not expect full quantitative agreement with our experimental results of NTCDA and PTCDA molecules in different adsorption geometries.

Figure 2(a) shows the calculated band structure of NTCDA on $\mathrm{Ag}(111)$ in the experimentally observed geometry. Due to the presence of the molecular monolayer, the period of the unit cell has grown in comparison with the clean $\mathrm{Ag}(111)$ surface, and the corresponding surface Brillouin zone has reduced its size. As a result, bands in the SBZ of the clean-surface unit cell get "folded" into the reduced SBZ. This leads to a surface band structure that no longer exhibits the projected band gap at the $\bar{\Gamma}$ point [cf. Fig. 1(b)]. As the (metal) electrons will actually have very small scattering probability at the border of this SBZ, this fact does not change the arguments given above for the decay of electrons excited into the IS, but it complicates the search for the IS in the calculated band structure. For that purpose we have analyzed the spatial probability distribution of states at different $\mathbf{k}$ points along symmetrical directions, averaged over the spatial coordinates $x y$ within the supercell (Fig. 2).

Characteristic for the IS is a probability density along the surface normal $\rho_{z}$ that resembles that of the Shockley state of bare $\mathrm{Ag}(111)$ in the silver film. It has a pronounced maximum in the region of the metal-organic interface and additional peaks around the center of the NTCDA molecules [Fig. 2(c)]. The dispersion of the IS reflects its metallic character and can be described by a free-electron like parabola [Fig. 2(a)]. Within our accuracy, the effective mass is independent of the geometry $\left(0.44 m_{\mathrm{e}}\right.$ for flat and $0.45 m_{\mathrm{e}}$ for bent molecules $)$ and close to that of the Shockley state. ${ }^{15}$ 
In contrast to the effective mass, the IS energy position at the $\bar{\Gamma}$ point is very sensitive to the exact geometry of the NTCDA adlayer [arrows in Fig. 2(a)]. For the distance of $2.997 \AA$, the bending only leads to a slight upshift of $41 \mathrm{meV}$, from 243 to $284 \mathrm{meV}$. The reduction of the distance by merely $5 \%$ to $2.85 \AA$, however, induces an upshift of $112 \mathrm{meV}$ to $355 \mathrm{meV}$. The, as compared to NTCDA, higher IS energy of the commensurate PTCDA layer can therefore to a large extent be explained by its slightly smaller adsorption distance.

Another important factor is the relative density of molecules per unit cell area in general and carbon rings in particular. Keeping only one NTCDA molecule in the unit cell, brings the IS energy $\sim 100 \mathrm{meV}$ closer to the Fermi level. This finding explains why, in spite of the shorter adsorption distance, the IS is observed at lower energy in the disordered, and consequently less densely packed LT-PTCDA phase. Furthermore, the previously calculated IS energy for PTCDA, with an adsorption distance of $2.85 \AA$, is $469 \mathrm{meV}$ at the $\bar{\Gamma}$ point. ${ }^{12}$ Although this value, corresponding to the bent geometry, should not be quantitatively compared with the $355 \mathrm{eV}$, obtained for flat NTCDA at $2.85 \AA$, the bending of the carboxyl groups is unlikely to be the dominating factor for the larger upshift of the PTCDA-IS. Rather this comparison suggests that, together with the reduction of the distance from the metal surface, the higher density of carbon rings per unit cell area can account for the experimentally observed energy difference of $200 \mathrm{meV}$ between the commensurate NTCDA and PTCDA layers. Both factors steepen the potential at the metal/organic as compared to the metal/vacuum interface and thus cause the Shockley state of the metal to shift to higher energies.

Whereas the bending of the carboxyl groups does not have a large effect on the IS energy position, it significantly changes the lateral probability distribution $\rho_{x y}$ of the wave function. In particular, the amplitude at the bridging oxygen atoms and at the carbon atoms of the carboxyl groups is greatly enhanced as compared to the flat molecule [Fig. 2(b)]. In addition, $\rho_{x y}$ is redistributed within the naphthalene core. Averaged over the whole SBZ, $\rho_{z}$ becomes larger on the molecule and diminished in the metal [Fig. 2(c)]. This explains the longer lifetime of the IS of LT-PTCDA compared to the ordered phase since NIXSW experiments have shown a significantly stronger bending of the carboxyl groups of these molecules toward the metal. ${ }^{16}$

In conclusion, we investigated the formation mechanism of unoccupied Shockley-derived metal-organic interface states. With adsorption of the PTCDA and NTCDA molecules on $\mathrm{Ag}(111)$ the Shockley state of the metal is shifted to energies above the Fermi level. The new energy position is primarily influenced by the presence of a $\pi$-electron system. It depends on adsorption distance and areal concentration of carbon rings. In contrast, the spatial probability density of the state, which has a pronounced maximum between the terminating silver atoms and the carbon plane, is mainly determined by the molecular geometry. Bending of the carboxylic end groups reduces the metallic and enhances the molecular overlap.

Funding by the Deutsche Forschungsgemeinschaft and the Ikerbasque Foundation is gratefully acknowledged.
${ }^{1}$ J. Hwang, A. Wan, and A. Kahn, Mater. Sci. Eng. R 64, 1 (2009).

${ }^{2}$ N. Koch, J. Phys. Condens. Matter 20, 184008 (2008).

${ }^{3}$ M. Rohlfing, R. Temirov, and F. S. Tautz, Phys. Rev. B 76, 115421 (2007).

${ }^{4}$ Y. Zou, L. Kilian, A. Schöll, T. Schmidt, R. Fink, and E. Umbach, Surf. Sci. 600, 1240 (2006).

${ }^{5}$ X. Y. Zhu, Surf. Sci. Rep. 56, 1 (2004).

${ }^{6}$ P. Tegeder, S. Hagen, F. Leyssner, M. V. Peters, S. Hecht, T. Klamroth, P. Saalfrank, and M. Wolf, Appl. Phys. A 88, 465 (2007).

${ }^{7}$ H. Yamane, D. Yoshimura, E. Kawabe, R. Sumii, K. Kanai, Y. Ouchi, N. Ueno, and K. Seki, Phys. Rev. B 76, 165436 (2007).

${ }^{8}$ N. Gonzalez-Lakunza, I. Fernandez-Torrente, K. J. Franke, N. Lorente, A. Arnau, and J. I. Pascual, Phys. Rev. Lett. 100, 156805 (2008).

${ }^{9}$ R. Temirov, S. Soubatch, A. Luican, and F. S. Tautz, Nature (London) 444, 350 (2006).

${ }^{10}$ A. Yang, S. T. Shipman, S. Garrett-Roe, J. Johns, M. Strader, P. Szymanski, E. Muller, and C. Harris, J. Phys. Chem. C 112, 2506 (2008).

${ }^{11}$ C. H. Schwalb, S. Sachs, M. Marks, A. Schöll, F. Reinert, E. Umbach, and U. Höfer, Phys. Rev. Lett. 101, 146801 (2008).

${ }^{12}$ N. L. Zaitsev, I. A. Nechaev, and E. V. Chulkov, Zh. Eksp. Teor. Fiz. 137, 129 (2010); J. Exp. Theor. Phys. 110, 114 (2010).

${ }^{13}$ M. S. Dyer and M. Persson, New J. Phys. 12, 063014 (2010).

${ }^{14}$ T. Andreev, I. Barke, and H. Hovel, Phys. Rev. B 70, 205426 (2004).
${ }^{15}$ F. Forster, S. Hüfner, and F. Reinert, J. Phys. Chem. B 108, 14692 (2004).

${ }^{16}$ L. Kilian, A. Hauschild, R. Temirov, S. Soubatch, A. Scholl, A. Bendounan, F. Reinert, T. L. Lee, F. S. Tautz, M. Sokolowski, and E. Umbach, Phys. Rev. Lett. 100, 136103 (2008).

${ }^{17}$ S. Sachs, C. H. Schwalb, M. Marks, A. Schöll, F. Reinert, E. Umbach, and U. Höfer, J. Chem. Phys. 131, 144701 (2009).

${ }^{18}$ A. Schöll, L. Kilian, Y. Zou, J. Ziroff, S. Hame, F. Reinert, E. Umbach, and R. H. Fink, Science 329, 303 (2010).

${ }^{19}$ T. Ozaki and H. Kino, Phys. Rev. B 72, 045121 (2005).

${ }^{20}$ U. Stahl, D. Gador, A. Soukopp, R. Fink, and E. Umbach, Surf. Sci. 414, 423 (1998).

${ }^{21}$ Unlike in the IR + UV excitation scheme employed in Ref. 11, the image-potential states do not contribute to the measured 2PPE intensity with blue + UV excitation.

${ }^{22} \mathrm{P}$. M. Echenique, R. Berndt, E. V. Chulkov, T. Fauster, A. Goldmann, and U. Höfer, Surf. Sci. Rep. 52, 219 (2004).

${ }^{23}$ J. J. Quinn and R. A. Ferrell, Phys. Rev. 70, 205426 (2004).

${ }^{24}$ W. Berthold, U. Höfer, P. Feulner, E. V. Chulkov, V. M. Silkin, and P. M. Echenique, Phys. Rev. Lett. 88, 056805 (2002).

${ }^{25}$ The peak shows an strong asymmetry toward higher energies, most likely due to contributions from stochastically formed small Herringbone areas. The evaluated position of the peak maximum shifts only little with emission angle, probably because parallel momentum in 2PPE is not conserved for the disordered layer.

${ }^{26}$ C. Stadler, S. Hansen, A. Schöll, T. L. Lee, J. Zegenhagen, C. Kumpf, and E. Umbach, New J. Phys. 9, 1 (2007). 